\title{
REFINING AND MAINTAINING THE OPTIMAL PERFORMANCE OF THE CEBAF SRF SYSTEMS *
}

\author{
C. Reece, J. Benesch, and J. Preble ${ }^{\dagger}$ \\ Thomas Jefferson National Accelerator Facility, Newport News, VA, 23606, USA
}

\begin{abstract}
CEBAF at Jefferson Lab is striving to achieve its maximum reliability at the maximum deliverable energy for physics experiments. Most critical are the acceleration systems. Characterizations of the individual limiting characteristics of the 338 superconducting rf (SRF) cavities continue to be refined. The field-emissionstimulated discharges at the cold $\mathrm{rf}$ waveguide input windows remain the dominant energy-limiting effect. By refining the cavity-specific dependences of this window arcing, we are able to minimize the resulting lost beam time when CEBAF is run near its maximum energy. Operation at increased rf power levels has produced unanticipated heating in the region near the warm polymeric rf windows on a few cavities. This has stimulated the migration to a more durable ceramic replacement window. During 2000, CEBAF encountered its first cryomodule helium leak into beam vacuum, most likely through an indium wire seal. Characterization of the leak and accommodation methods used will be described. Finally, in situ $\mathrm{rf} / \mathrm{helium}$ processing of cavities continues to increase the available linac voltage.
\end{abstract}

\section{INTRODUCTION}

While planning and preparations are underway for an upgrade of CEBAF to $12 \mathrm{GeV}$,[1] efforts persist toward exploiting the full capability of the present installation. The SRF systems continue to be quite reliable, rarely showing signs of degradation. The desire to support physics experiments at energies approaching $6 \mathrm{GeV}$ forces attention toward making incremental improvements and continued refinement of the parametrization of the performance limits of each of the 338 SRF cavities and associated controls. Some modest maintenance activities aim to keep the present cryomodules operational for the foreseeable future.

\section{WINDOW ARCING}

Arcing at the cold ceramic window is driven by field emission in cavities. The field emission is generally well described by the Fowler-Nordheim tunneling model or by

\footnotetext{
*Work supported by the U.S. Department of Energy, contract DE-AC05-84ER40150

${ }^{\dagger}$ reece, benesch, preble@jlab.org
}

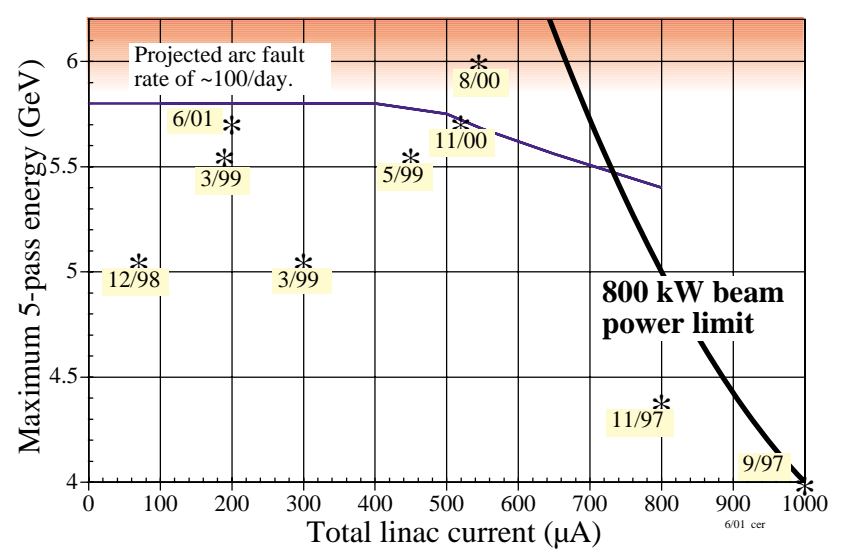

Figure 1. Highpoints of CEBAF beam an empirical model exponential in gradient.[2] The key assumptions needed to apply these models are:

- Arc faults occur when a fixed charge is accumulated on the cold ceramic window.

- The value of this charge is unchanged over years.

- The charge is reset to the same low value after each arc.

- The ceramic window has very low leakage current.

- The clock intervals between faults may be approximately corrected to include only periods when rf was on at a constant operating gradient $(G)$.

- The charge accumulation rate is proportional to the field-emitted current from a single dominant emitter.

These allow one to write:

Charge $=$ constant $=j^{*}$ (corrected fault interval)

$j=$ constant $/$ (corrected fault interval)

Plotting $\ln \left(1 /\left(\right.\right.$ interval $\left.\left.* G^{2}\right)\right)$ vs. $1 / G$ should then result in a straight line if the Fowler-Nordheim model holds. If the empirical exponential model holds, $\ln (1 /$ interval $)$ vs. $G$ should be a straight line. Such Fowler-Nordheim plots have been created for each cavity and the data.[3] The resulting models of fault interval as a function of gradient on average explain about half the variation in fault interval. The portion of variation explained by statistically significant models ranges from $10-95 \%$.

There are 315 usable cavities in the two linacs. Of these, statistically significant $(p=0.05)$ models for arcing have been obtained for 211. Since the exponential model can be solved in closed form while the Fowler-Nordheim model cannot, the former was implemented in a program to optimize accelerator setup, lem++.[4] This program takes as input the gradients at which cavities trip at 8 hour 
intervals and the slopes of the $\ln (1 /$ interval $)$ vs. $G$ fits. Histograms of these values are shown in Figure 2.
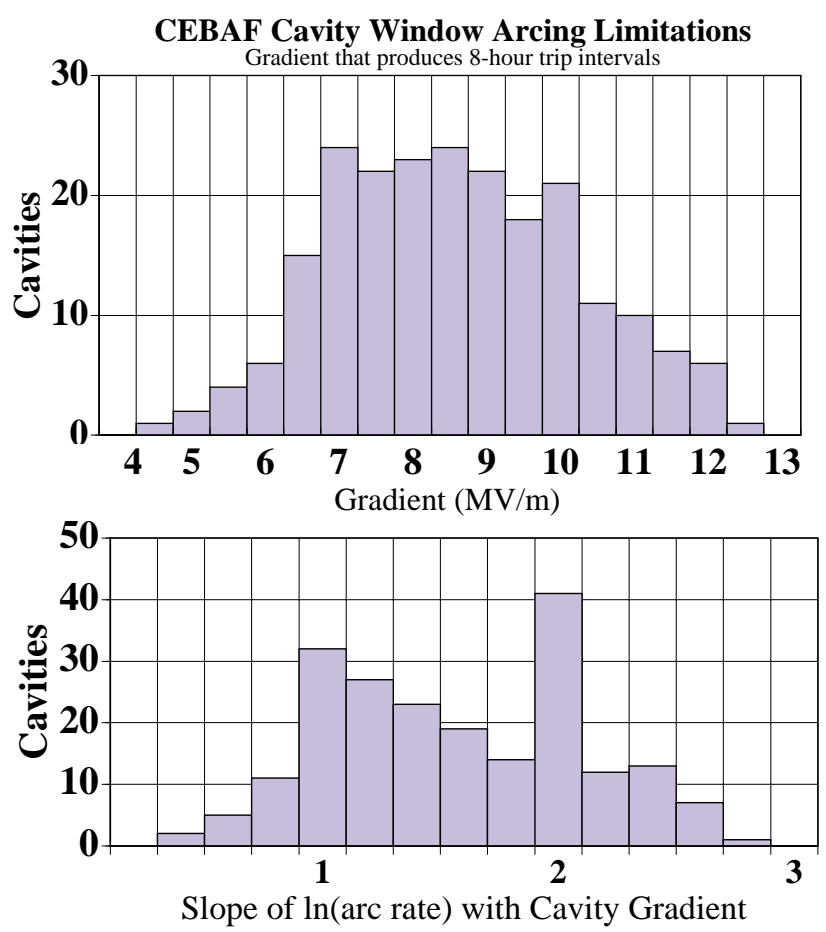

Figure 2. Distribution of 8-hour arc gradients and slopes

The mean of the slope distribution is equivalent to a factor of 5 change in fault rate for a gradient increase of $1 \mathrm{MV} / \mathrm{m}$. (A slope of 2 is used as default with sparse data.)

As discussed in [4], one wants to operate the machine at the lowest fault rate consistent with available cooling and $\mathrm{RF}$ power, the two biggest non-personnel costs for accelerator operation. Our refrigeration system can provide $1 \mathrm{~W}$ at $2 \mathrm{~K}$ for $1200 \mathrm{~W}$ at room temperature; it draws $\sim 5 \mathrm{MW}$. RF power is limited more by tube life than the power bill: running at rated voltage during a $6 \mathrm{GeV}$ test in August 2000, we lost one klystron per day. Running at $90 \%$ of this voltage, we haven't lost any in eight months. 109 probe tuners have been installed on the feed waveguides of better cavities to improve the match of the klystron to the beam. Matching cannot be fully optimized due to Lorentz detuning effects that cannot be managed by the present RF controls. Arc rates as a function of energy for the machine setup as of 1 June 2001 were predicted using lem++. These are plotted for linac beam currents of 300 and $600 \mu \mathrm{A}$ in Figure 3 .

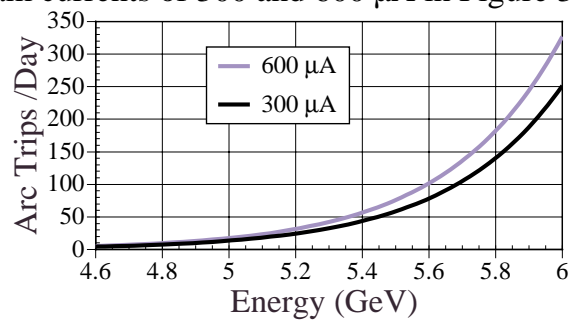

Figure 3. Arc trip frequency with CEBAF 5-pass energy
In the initial setup of CEBAF, little tolerance was allowed for field emission loading. As the energy requested by users increased, this low tolerance has been increasingly relaxed. Cavities that did not arc were pushed farther into field emission. Initially, there were no problems. During the first physics run at $5.73 \mathrm{GeV}$, a phenomenon which had previously been anecdotal prior to this time became a dominant factor in arc rate: cavities whose arcs are driven not by their own field emission, but rather by field emission in neighboring or even nextnearest neighbor cavities.[3] Some 20 of these were identified, some by statistical analysis and some empirically, and suspects were turned down. This cut the rather objectionable arc rate in half. The specific mechanism is not understood. Victims are easily identified: many arcs with no gradient dependence. Finding suspects is harder since there is a clear case where one cavity drove arcs in the two cavities upstream of it. Investigation continues. At best, there is a clear turn-on date of the different behavior of the victim and an associated substantial increase in the gradient of a nearby suspect. Searching through the data available to look for such correlations is tedious.

\section{COLD HELIUM LEAK}

During the August 2000 maintenance down, the room temperature waveguide windows on cryomodule NL11 were replaced. Anomalous heating had been observed on the flanges adjoining the windows, and there was concern about eventual window loss of integrity. This window maintains the vacuum in the thermal transition region of the input waveguide. The module was warmed to room temperature and the windows exchanged. After cooldown, some minor problems were encountered with rf controls on two of the eight cavities, but otherwise the module appeared to function normally.

Over the subsequent several weeks, however, the cavities became progressively unusable. The rf controls behaved erratically. A systematic verification of control hardware and $\mathrm{rf}$ waveguide components found no problems.

The poor performance was eventually diagnosed as the result of a cold helium leak into the beamline, somewhere in the region of the first cavity pair. Presumably the leak was opened by the thermal cycle to room temperature. Since several weeks of useful operation had been obtained before the onset of problems, we warmed the module to $\sim 30 \mathrm{~K}$ and pumped out the beamline in the same manner as done following helium processing. This minimal "burping" of the module entails minimal additional risk and has been effective at restoring NL11 to service for periods of several weeks. When the module is needed for high energy running, it is now considered routine maintenance to "burp" it approximately every five weeks. This is accomplished during a maintenance period and requires about 18 hours from $\mathrm{rf}$ off to module full and $\mathrm{rf}$ 
back on. Based on the occurrence of similar rf behavior during deliberate helium processing, the cold superfluid leak rate is estimated to be of order $2 \mathrm{e}-5 \mathrm{std} \mathrm{cc} / \mathrm{s}$ compared with a mean superfluid helium leak rate of $4 \mathrm{e}-11 \mathrm{std} \mathrm{cc} / \mathrm{s}$ per cavity pair measured in vertical test.[5]

\subsection{WARM RF WINDOW ISSUES}

During the life of CEBAF we have lost several polyethylene windows as a result of rf heating melting the window. In each case an uncontrolled loss of vacuum in the waveguide has resulted. Recovery has been possible in each case but has required the warming of the cryomodule to room temperature, removing the old window, cleaning the waveguide vacuum space, installing a new window, and cooling the cryomodule back down to $2 \mathrm{~K}$. This recovery typically takes a minimum of one week and has, in some cases, compromised cavity performance. In all but two cases an rf/cavity interlock had been defeated, and challenging circumstances resulted in excessive rf heating and window failure. In the remaining two cases all rf/cavity interlocks were in a working state. Anomalous rf flange heating during operation at increased forward power levels, however, threatened the window integrity. In these instances the IR interlock was expected to have prevented these failures by inhibiting rf power. A failure mode has been identified where a slow heating of the entire warm window assembly area can effectively blind the IR detector, reducing its sensitivity to increased window temperature.

In one instance we have lost the vacuum integrity of a warm window without catastrophic melting. This window, an early version, was made of Teflon, which has a considerably higher melting temperature. In this case the window appeared to crack instead of melt, resulting in a vacuum leak that was controlled before warm-up of the cryomodule was required. It is known that Teflon has limited radiation hardness, and we may be significantly "aging" these windows in the accelerator environment. The cavity associated with this window is inoperable until warm-up and window replacement is accomplished. Presently there are seven cryomodules with a total of 56 Teflon windows installed in the accelerator.

We have designed a replacement for this warm $\mathrm{rf}$ window on the first-generation CEBAF cryomodule. The window is a modification of an existing window design developed for the CEBAF upgrade and FEL application. The window uses a ceramic window brazed into a kovar cup that is in turn brazed into a stainless steel flange. This assembly is bolted onto the existing cryomodule warm window assembly using an indium wire seal. Two cryomodules, one in the FEL and one in CEBAF slot SL21, have been operating with similar windows for considerable operational hours at power levels up to $30 \mathrm{~kW}$.

We are developing a new technique for changing warm windows on installed cryomodules that does not include a full warm-up. In order to change the warm window, the waveguide vacuum space must be vented. This has traditionally involved warming the cryomodule to room temperature to avoid condensation of air products at the cavity interface. The current plan is to vent and purge the waveguide vacuum space with helium while maintaining the cryomodule at $\sim 40 \mathrm{~K}$. At this temperature helium will not condense and can be pumped out after changing of the window. A test of our ability to maintain a cryomodule in this temperature range was performed during the May 2001 CEBAF maintenance period. The module was maintained at an intermediate temperature for several days, but was accidentally allowed to warm to $\sim 70 \mathrm{~K}$, well above the target temperature. Additional testing will be required to qualify the change-out technique. Installation of the first replacement windows is now scheduled for September 2001.

\section{IN SITU HELIUM PROCESSING}

The only means known for improving the performance of installed field-emission-limited SRF cavities is helium/rf processing. Between September 1996 and January 2000, 38 of the 42 cryomodules in CEBAF were processed in this way. The procedure was refined with use. In some of the early cryomodule processing, obtainable gradients were frequently limited by waveguide vacuum instabilities. Subsequent procedure revisions deliberately degas the waveguides prior to the processing, allowing operation at higher gradients and thus more effective helium processing. Approximately $78 \mathrm{MV} /$ pass of additional usable voltage was obtained.

In May 2001, four previously processed cryomodules were reprocessed to assess whether a complete second pass at processing would be beneficial. Two modules that had received early processing in 1997 yielded an additional $8 \mathrm{MV}$, so further attention will be given to several more cryomodules that received early treatment. Further such progress may enable operations at $6 \mathrm{GeV}$.

\section{REFERENCES}

[1] http://www.jlab.org/div dept/physics division/GeV.html

[2] R. J. Noer, App. Phys A 28, 1-24 (1982), and

J. F. Benesch, "Statistical Analysis of RF Cavity Faults", IEEE Trans. Applied Superconductivity vol. 9 \#2, June 1999, p. 888

[3] "Statistical Analysis of Cavity Arc Faults - April 2001

Status", JLab-TN-01-020, J. Benesch at http://tnweb.jlab.org/tn/2001/01-020.pdf

[4] J. R. Delayen et al., "Operational Optimization of Large Scale SRF Accelerators," Proc. of 1999 Particle Accelerator Conf. , 940 (1999)

[5] J. F. Benesch and C. E. Reece, "CEBAF's SRF Cavity Manufacturing Experience", Advances In Cryogenic Engineering vol 39, p. 597, ed. P. Kittel (Plenum Press, 1994) 